\title{
Obstáculos para la movilidad académica con el programa Erasmus+: Diferencias entre grados universitarios Barriers to academic mobility with Erasmus+ programme: Differences between bachelor degrees
}

\author{
Fajó-Pascual $\mathrm{M}^{1}$, Sanclemente $\mathrm{T}^{1}$, Molina $\mathrm{A}^{2}$, Asún $\mathrm{S}^{1}$, Romero $\mathrm{R}^{1}$, Poblador $\mathrm{JA}^{1}$, Ferrer-Mairal $\mathrm{AM}^{1}$, Gómez $\mathrm{EM}^{1}$, \\ Vercet $\mathrm{A}^{1}$ \\ mfajo@unizar.es, tsanclem@unizar.es,606506@unizar.es, sonasun@unizar.es,rromero@unizar.es,pobla@unizar.es, \\ ferrerma@unizar.es, evagomez@unizar.es, vercet@unizar.es \\ ${ }^{1}$ Facultad de Ciencias de la Salud y del Deporte \\ Universidad de Zaragoza \\ Huesca, España \\ ${ }^{2}$ Facultad de Ciencias de la Salud \\ Universidad de Zaragoza \\ Zaragoza, España
}

\begin{abstract}
Resumen- Pese al interés creciente que presenta la movilidad académica internacional de los estudiantes universitarios europeos para su inserción profesional posterior, el porcentaje de estudiantes españoles que participan en el programa Erasmus es todavía pequeño. El objetivo de este trabajo fue determinar los principales obstáculos para la movilidad Erasmus percibidos por estudiantes de grado que no han participado en este programa, valorando la existencia de diferencias en función del grado (Sanitario vs. Ciencias Sociales). Se utilizó una encuesta auto-administrada elaborada para un estudio europeo previo en el que participó España. Los resultados muestran el impacto, a nivel general, de la falta de información sobre temas relevantes como: la calidad de la educación en el extranjero, o cómo van a ser evaluados; así como, las barreras económicas por becas insuficientes y retrasos en el pago de las mismas. Los obstáculos que mejor diferencian a los estudiantes de ambos grados están relacionados con la ordenación académica del grado, en concreto, bajo nivel de formación en inglés y presencia de asignaturas anuales, además de características personales del estudiante. Para incrementar la participación, se debería incidir en informar más y comunicar mejor además de incluir modificaciones en el programa académico de los grados que faciliten la movilidad internacional.
\end{abstract}

Palabras clave: Obstáculos, No participantes, Movilidad académica, Grados, Erasmus, Internacionalizacion

Abstract- Despite the growing interest that presents academic international mobility for European University students for their later in life job prospects, the rate of Spanish students participating in the Erasmus programme is still small. The objective of this research was to determine the main barriers perceived by bachelor students who had not participated in the Erasmus programme assessing the differences between two bachelor degrees (Health vs. Social Sciences). A self-administered survey including reasons not to participate was used that had been set up for a previous European study including Spain. Results reveal the overall impact of lack of information on relevant issues such as the quality of university education system abroad and how they will be evaluated as well as financial barriers such as insufficient Erasmus grants or delays in the first grant payment. The barriers that better differentiate both bachelor degrees students are related to the bachelors' curriculum i.e. insufficient training in English and presence of annual (vs. semester) courses as well as personal features. To increase participation, the focus should be on improving information and communication both on academic and financial issues, and changes in the bachelors' curricula.

Keywords: Barriers, Non-participants, Academic mobility, Bachelors, Erasmus, Internationalization

\section{INTRODUCCIÓN}

Este estudio profundiza en las razones percibidas por estudiantes universitarios en España para no participar en una movilidad académica Erasmus, además de valorar posibles diferencias en la relevancia de los obstáculos percibidos en función del ámbito de estudios de los estudiantes.

Los resultados de este estudio permitirán informar el establecimiento de prioridades de actuación de los agentes implicados en el apoyo de la movilidad internacional Erasmus, a nivel de grado, Facultad/Universidad y organismos con competencias en educación superior (Gobierno autónomo y central).

\section{CONTEXTO}

Se ha señalado que la movilidad académica internacional de los estudiantes presenta beneficios tanto a nivel individual, en relación a su desarrollo personal y su inserción profesional, como también, a nivel institucional, contribuyendo a la internacionalización de las Universidades. De igual modo, se considera como un indicador de calidad en ránquines mundiales (Souto-Otero, Huisman, Beerkens, de Win \& Vujic, 2013).

A pesar de esta evidencia, la movilidad internacional solo alcanza a una minoría de estudiantes universitarios europeos (<4\%) (Vossensteyn et al, 2010); y eso, aun cuando en el Espacio Europeo de Educación Superior (EEES) se han llevado a cabo reformas estructurales con el proceso de Bolonia y se han puesto en marcha programas de movilidad apoyados económicamente, cuyo ejemplo más destacado en Europa es el programa Erasmus. 
La Estrategia de Movilidad para un Mejor Aprendizaje, acordada en la conferencia ministerial de la UE en Bucarest 2012 para el proceso de Bolonia, proponía acciones para que los países miembros alcanzaran el objetivo fijado ya en el Comunicado Ministerial de Lovaina de 2009 de que "en el año 2020 al menos un 20\% de los graduados en el EEES hubiera tenido un periodo de estudios o de prácticas en el extranjero" (EHEA, 2012).

El porcentaje de alumnos universitarios españoles que realizó una movilidad académica internacional con el programa Erasmus (Erasmus+ desde 2014) durante el curso académico 14-15, rondó el 2,5\% (Ministerio de Educación, Cultura y Deporte, 2014). Existe, por tanto, un importante margen para la mejora pues el alcance de esta experiencia es por el momento limitado.

La no participación en la movilidad académica Erasmus+ parece ser más un proceso de autoexclusión por parte de los estudiantes, que de selección por parte de las instituciones, como sugiere Teichler (2004). Por ello, es importante conocer los obstáculos a los que se enfrentan aquellos estudiantes que deciden no participar, pues se ha detectado que la importancia de esas barreras parece cambiar de país a país (Vossensteyn et al, 2010).

Además, existen importantes diferencias en participación en función del ámbito de estudio, siendo las titulaciones de ciencias sociales (CCSS), empresariales y derecho los que presentan un mayor porcentaje de movilidad, frente a ciencias de la salud y servicios, que ocuparía un $4^{\circ}$ lugar según datos del 2013-14 (MECD, 2016).

La tasa de participación de los estudiantes de un grado en CCSS de nuestra Facultad, aun siendo variable de año en año, ha mostrado un mejor comportamiento que la de un grado Sanitario (S) impartido en el mismo centro, como pusieron de manifiesto los autoinformes de evaluación de la calidad elaborados con motivo de su reciente acreditación en 2016.

El objetivo de este estudio fue determinar los principales obstáculos a la movilidad internacional percibidos por los estudiantes que no habían realizado una movilidad académica internacional con el programa Erasmus en nuestro centro, valorando si difieren en su relevancia en un grado universitario de cuatro años en el ámbito Sanitario y otro, en Ciencias Sociales.

\section{DESCRIPCIÓN}

Se utilizó un diseño descriptivo transversal mediante encuesta auto-administrada en clase.

La Facultad en la que se realizó el estudio cuenta con aproximadamente 750 alumnos matriculados, localizándose en un campus periférico de una Universidad de tamaño medianogrande. El número de estudiantes matriculados en el curso 1617 en el grado S fue de 248, frente a 292 en el grado de CCSS. En el primero, un 69\% (171) de las matriculadas fueron mujeres, mientras que en el segundo, la cifra fue de un $22 \%$ (63).

En el grado $\mathrm{S}$ se ofertaban como destinos, 10 universidades en 8 países con 23 plazas y en el de CCSS, 13 universidades en 6 países con 32 plazas. Todos los destinos del grado $\mathrm{S}$ requerían al menos un nivel B1 de inglés del MCER. Un destino del grado en CCSS no requería certificación de nivel de idioma inglés ni portugués y para varios destinos franceses, se satisfacía el nivel de idioma requerido con haber superado la asignatura de francés en $4^{\circ}$ de la ESO o haberla cursado al menos un año en bachillerato. La mayor parte de las estancias académicas internacionales Erasmus de los alumnos de CCSS son anuales, mientras que en el caso de los estudiantes del grado S, la mayor parte de los destinos sólo permiten estancias semestrales.

La encuesta se distribuyó de forma presencial entre los meses de marzo y mayo del 2017, en una asignatura de cada uno de los cuatro cursos de los dos grados, en la que se presumía mayor asistencia

La encuesta se diseñó a partir de otra, que había sido utilizada previamente en un estudio realizado en varios países europeos incluyendo España, donde se recogía entre otra información, los obstáculos para la No participación de aquellos universitarios que no habían llevado a cabo una movilidad académica Erasmus (Souto-Otero, 2013). La encuesta estuvo disponible en varias lenguas incluido el español. A esas barreras se añadieron otras relacionadas con el plan de estudios propio de los grados seleccionados.

La encuesta estuvo compuesta por 30 items organizados en 5 grupos: Conocimiento/información sobre el programa de movilidad Erasmus, Características personales del estudiante, Dificultades de tipo económico, Complejidad intrínseca del programa de movilidad Erasmus, Características del grado y Comparabilidad de sistemas educativos universitarios en Europa.

Se valoraba la importancia de los mismos como obstáculos para la movilidad académica internacional Erasmus+ en una escala Likert de 1 a 5, en la que 1= Nada importante y 5= Muy importante.

La encuesta recogía también información sociodemográfica como el género, la edad y el nivel de estudios de padre y madre, así como información académica en relación al grado universitario, como qué grado, curso académico, nota acceso, sistema de acceso, elección primera opción, además de conocimientos previos de idioma inglés certificado y percibido. Además, se les preguntó si habían realizado una movilidad académica con el programa Erasmus+, y a los que respondieron negativamente, que fueron los estudiantes incluidos en nuestro estudio, se les preguntó adicionalmente si estarían interesados en solicitarla durante el grado.

Se realizó un análisis estadístico descriptivo resumiendo las variables cuantitativas, como medias y desviaciones típicas (DT), o medianas y rango intercuartílico, teniendo en cuenta su normalidad; mientras que las variables categóricas se resumieron mediante recuentos y porcentajes. Las variables de escala se dicotomizaron en $0=$ No importante y $1=$ Importante si se había respondido de 1-3 y de 4-5 respectivamente.

En cuanto al análisis inferencial, para la comparación de proporciones se utilizó el test de chi cuadrado o el exacto de Fisher (si no se cumplían condiciones de aplicación del anterior), mientras que para la comparación de variables cuantitativas se empleó el test $\mathrm{T}$ de Student o U de MannWhitney (si no se cumplían condiciones de aplicación del anterior). 
Se utilizó SPSS versión 22 como software estadístico. Todos los contrastes fueron bilaterales y el nivel de significación fue del 5\%.

\section{RESUltados}

El número de alumnos que rellenó la encuesta fue de 301 (196 CCSS y 105 S). La tasa de respuesta a nivel general fue del $55,7 \%$ y por grados $67,1 \%$ y $42,3 \%$ para los grados de CCSS y S, respectivamente.

La descripción estadística de la muestra se encuentra en Tabla 1. La edad mediana fue de 21 años con un 41,9\% de mujeres. La mayoría se encontraban en $2^{\circ}$ curso o superior (63,1\%), y habían accedido al grado con un bachillerato (74,1\%) con una nota mediana de 9,6. Un 80,1\% había elegido su grado como $1^{\circ}$ opción.

Tabla 1

Características sociodemográficas y académicas de estudiantes de grado que no habían realizado una movilidad académica internacional Erasmus+

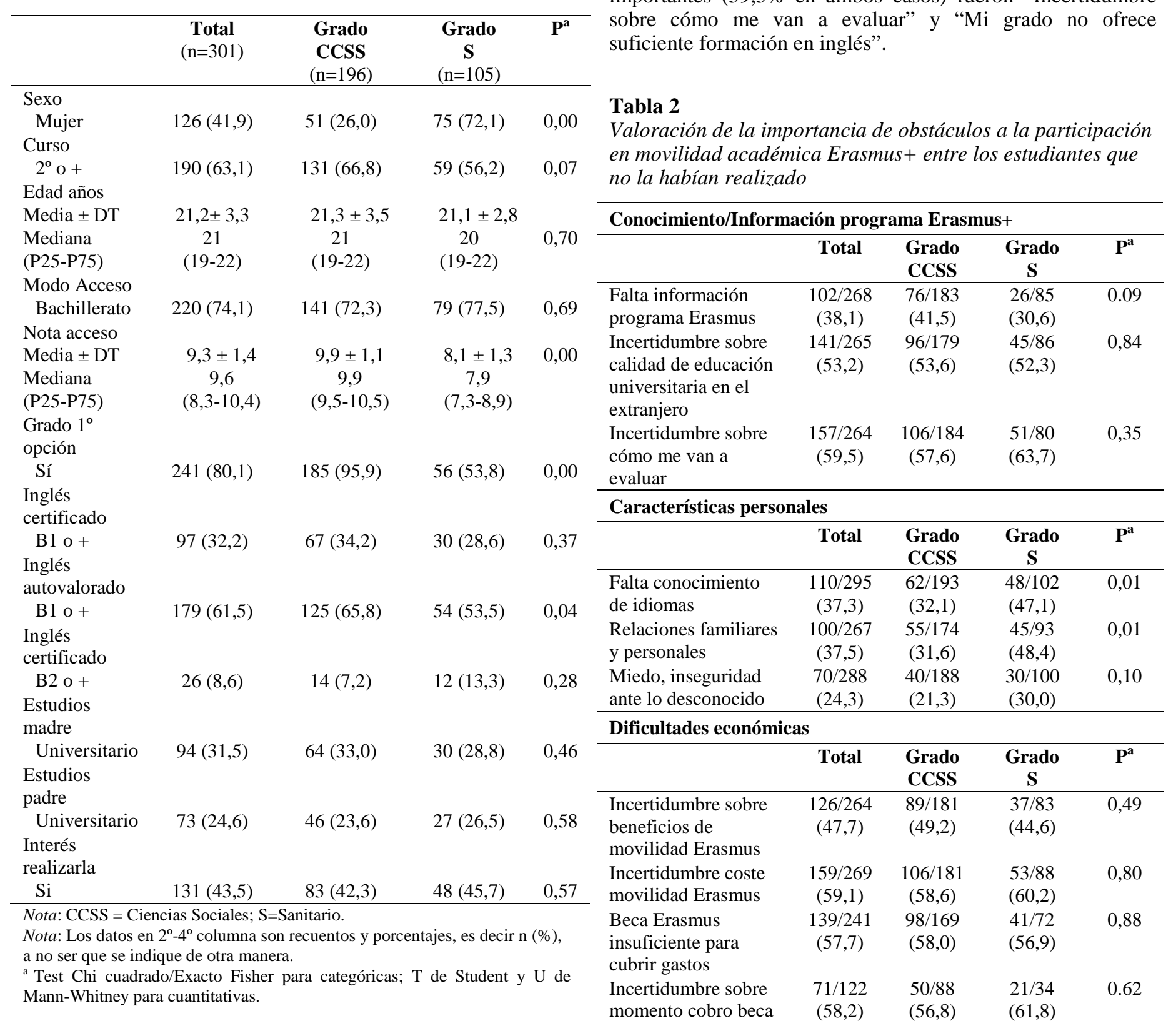

Octubre 4-6, 2017, Zaragoza, ESPAÑA

IV Congreso Internacional sobre Aprendizaje, Innovación y Competitividad (CINAIC 2017)

Un 32,2\% contaba con un nivel B1 inglés certificado, mientras que un nivel B2 tan sólo lo reportaba un 8,2\%. En un $31,5 \%$ de los casos, la madre poseía estudios universitarios. Un 43,5\% de ellos, pese a que no habían participado en el programa Erasmus, tenían todavía interés en hacerlo.

La comparación de características por grados (CCSS vs Sanitario) se muestra también en la Tabla 1. El porcentaje de mujeres en el grado sanitario fue significativamente superior (72,1\% vs $26,0 \%$ ). La nota media y mediana de ingreso fue significativamente superior en el grado de CCSS (1,8 y 2 puntos sobre 10 por encima), así como el porcentaje de estudiantes que eligieron este grado como $1^{\circ}$ opción (95,9 vs $35,8 \%)$. El porcentaje de alumnos en $2^{\circ}$ curso o superior fue mayor en el grado de CCSS, pero al límite de la significación estadística (66,8 vs. 56,2, p=0,07).

La valoración de los ítems de la encuesta expresada en forma de porcentaje de estudiantes que lo consideró importante se encuentra en Tabla 2. En conjunto, los ítems que fueron más frecuentemente considerados como obstáculos importantes $(59,5 \%$ en ambos casos) fueron "Incertidumbre sobre cómo me van a evaluar" y "Mi grado no ofrece

\section{Tabla 2}

Conocimiento/Información programa Erasmus 
Elevada competencia por becas Erasmus+

$95 / 226$

$60 / 158$

$(42,0)$

$(38,0)$

35768

$(51,5)$

0,06 complementarias

Características intrínsecas del programa Erasmus+

\begin{tabular}{|c|c|c|c|c|}
\hline & Total & $\begin{array}{l}\text { Grado } \\
\text { CCSS }\end{array}$ & $\begin{array}{c}\text { Grado } \\
\text { S }\end{array}$ & $\mathbf{P}^{\mathbf{a}}$ \\
\hline $\begin{array}{l}\text { Complejidad } \\
\text { administrativa del } \\
\text { programa Erasmus+ }\end{array}$ & $\begin{array}{l}68 / 217 \\
(31,3)\end{array}$ & $\begin{array}{l}51 / 157 \\
(32,5)\end{array}$ & $\begin{array}{l}17 / 60 \\
(28,3)\end{array}$ & 0,55 \\
\hline $\begin{array}{l}\text { La movilidad } \\
\text { académica retrasaría } \\
\text { momento graduación }\end{array}$ & $\begin{array}{c}84 / 237 \\
(35,4)\end{array}$ & $\begin{array}{c}55 / 163 \\
(33,7)\end{array}$ & $\begin{array}{l}29 / 74 \\
(39,2)\end{array}$ & 0,42 \\
\hline \multicolumn{5}{|l|}{ Características grado } \\
\hline & Total & $\begin{array}{l}\text { Grado } \\
\text { CCSS }\end{array}$ & $\begin{array}{c}\text { Grado } \\
\text { S }\end{array}$ & $\mathbf{P}^{\mathbf{a}}$ \\
\hline $\begin{array}{l}\text { Mi grado no ofrece } \\
\text { suficiente formación } \\
\text { en inglés }\end{array}$ & $\begin{array}{c}166 / 279 \\
(59,5)\end{array}$ & $\begin{array}{c}121 / 187 \\
(64,7)\end{array}$ & $\begin{array}{l}45 / 92 \\
(48,9)\end{array}$ & 0,01 \\
\hline $\begin{array}{l}\text { Asignaturas anuales } \\
\text { en mi grado (si } \\
\text { estancia semestral) }\end{array}$ & $\begin{array}{c}101 / 231 \\
(43,7)\end{array}$ & $\begin{array}{c}59 / 156 \\
(37,8)\end{array}$ & $\begin{array}{l}42 / 75 \\
(56,0)\end{array}$ & 0,01 \\
\hline
\end{tabular}

\begin{tabular}{|c|c|c|c|c|}
\hline \multicolumn{5}{|c|}{ Comparabilidad de sistemas educativos universitarios Europa } \\
\hline & Total & $\begin{array}{l}\text { Grado } \\
\text { CCSS }\end{array}$ & $\begin{array}{c}\text { Grado } \\
\text { S }\end{array}$ & $\mathbf{P}^{\mathbf{a}}$ \\
\hline $\begin{array}{l}\text { Dificultad } \\
\text { establecimiento de } \\
\text { equivalencias entre } \\
\text { asignaturas (antes } \\
\text { salir) }\end{array}$ & $\begin{array}{c}76 / 172 \\
(44,2)\end{array}$ & $\begin{array}{c}51 / 126 \\
(40,5)\end{array}$ & $\begin{array}{l}25 / 46 \\
(54,3)\end{array}$ & 0,10 \\
\hline $\begin{array}{l}\text { Problemas con el } \\
\text { reconocimiento de } \\
\text { asignaturas en mi } \\
\text { Universidad (vuelta) }\end{array}$ & $\begin{array}{l}79 / 169 \\
(46,7)\end{array}$ & $\begin{array}{l}59 / 127 \\
(46,5)\end{array}$ & $\begin{array}{l}20 / 42 \\
(47,6)\end{array}$ & 0,90 \\
\hline $\begin{array}{l}\text { Falta apoyo en la } \\
\text { Universidad Acogida }\end{array}$ & $\begin{array}{l}71 / 180 \\
(39,4)\end{array}$ & $\begin{array}{l}52 / 131 \\
(39,7)\end{array}$ & $\begin{array}{l}19 / 49 \\
(38,8)\end{array}$ & 0,91 \\
\hline
\end{tabular}

Nota: CCSS = Ciencias Sociales; $\mathrm{S}=$ Sanitario.

Nota: Un obstáculo se consideró como importante si se respondió 4-5 en escala 1-5.

Nota: Los datos en la tabla son recuentos de respuestas que consideraron un ítem como importante sobre total de respuestas; entre paréntesis, ese cociente expresado como porcentaje (\%)

${ }^{a}$ Test Chi cuadrado o Exacto Fisher

Estos obstáculos fueron seguidos en orden por otros de índole económica como "Incertidumbre sobre el coste de una estancia académica Erasmus" (59,1\%), "Incertidumbre sobre el momento en que cobraré la beca” (58,2\%), y "Beca Erasmus insuficiente para cubrir los gastos adicionales que se generan” (57,7\%).

En $6^{\circ}$ lugar se señaló la "Incertidumbre sobre la calidad de la educación universitaria en el extranjero", seguido de "Incertidumbre sobre beneficios de una estancia académica Erasmus" valorados como importantes por 53,2\% y 47,7\% de los encuestados, respectivamente. En $8^{\circ}$ y $9^{\circ}$ lugar se sitúan ítems relacionados con el reconocimiento de asignaturas tras la movilidad y antes de la partida, valorados como importantes por un $46,7 \%$ y $44,2 \%$, respectivamente.

En la comparación de ambos grados, se observó una diferencia estadísticamente significativa a favor del grado $\mathrm{S}$ en la valoración como obstáculos importantes de los ítems "Falta de conocimiento de idiomas", "Relaciones familiares y personales" y "Existencia en el grado de asignaturas de carácter anual”, que fueron valorados como importantes por un $47,1 \%, 48,4 \%$ y $56,0 \%$ de los estudiantes $\mathrm{S}$, respectivamente, frente a un 32,1\%, 31,6\% y un 37,8\% por los estudiantes de CCSS.

Por el contrario, el ítem "Mi grado no ofrece suficiente formación en inglés" fue valorado más frecuentemente como importante por los estudiantes de CCSS (64,7\% vs 48,9\%, $\mathrm{p}=0,012$ ).

\section{CONCLUSIONES}

El porcentaje de participación de mujeres en nuestra encuesta fue similar al porcentaje de mujeres matriculadas en ambos grados, esto es, un $72 \%$ respuesta frente a un $69 \%$ de matrícula en el grado S, y un $26 \%$ respuesta frente a un $22 \%$ matricula, en el grado de CCSS. El porcentaje de mujeres que respondieron la encuesta en el grado $S$ fue casi 3 veces superior comparado con el grado en CCSS. Estos datos están en consonancia con el porcentaje de matrícula de mujeres en ambos grados, así como con lo reportado por Briz-Ponce, Juanes-Méndez y García-Peñalvo (2016) al describir la tendencia al alza en los últimos años del número de mujeres que cursan grados sanitarios en España y en otros países occidentales.

Los principales obstáculos para la movilidad que puso de manifiesto nuestro estudio, y que fueron señalados por alrededor de un $60 \%$ de los encuestados, tuvieron que ver con la información con la que cuenta el estudiante sobre el sistema educativo universitario en Europa, además de con la escasez de oportunidades de formación en idioma inglés que proporcionaron los programas académicos de los grados.

El $1^{\circ}$ obstáculo "Incertidumbre sobre el coste de una estancia académica Erasmus” y el $6^{\circ}$ "Incertidumbre calidad de la universidad en el extranjero" sugieren que al alumno se le plantean un buen número de dudas en el proceso de decisión ante una movilidad académica Erasmus. Podríamos concluir que el estudiante precisa más información, pero también, mejor comunicación de la misma de modo que se le ayude a hacer oscilar la balanza del lado de los beneficios que esta experiencia puede reportarle. No en vano, casi un 50\% de ellos señala no tener claro los beneficios que esta actividad puede proporcionarles.

En cuanto al segundo obstáculo, su percepción de que la insuficiente formación en inglés proporcionada por su grado sea un obstáculo para la movilidad académica internacional, estaría relacionada con el hecho de que en la selección para la movilidad, el contar con un nivel B1 del MCER de idioma inglés certificado, es en nuestro centro un requisito para la adjudicación de un destino, además de un mérito valorable.

En nuestro estudio solo un 32,2\% de los estudiantes contaba con ese nivel de competencia en inglés certificado, y si hablamos de un nivel B2, esa proporción se reduce a un 8,2\%. Además, el número de universidades europeas que exigen un nivel B2 del MCER en inglés, para aceptar estudiantes Erasmus entrantes, aumenta de año en año. La importancia de ese obstáculo fue significativamente más relevante para los estudiantes de CCSS, lo que podría explicarse porque su programa de estudios, a diferencia del programa del grado $\mathrm{S}$, no ofrece una asignatura dirigida a la enseñanza de este idioma.

Resulta llamativo que la "falta de conocimiento de idiomas de los estudiantes” adquiera por el contrario, mayor relevancia para los del grado S que para los de CCSS (47,1 vs 32,1), lo 
que se podría entender por la oferta de un mayor número de destinos Erasmus con exigencia de nivel B1 y B2 de inglés. Adicionalmente, un $30 \%$ de los estudiantes S comunicaba "Miedo, inseguridad frente a lo desconocido" como obstáculo, frente a un 21,3\% de los de CCSS. Se podría interpretar porque las posibilidades de movilidad académica que se ofrecen a los estudiantes S son más exigentes en términos de idioma (posible contacto con pacientes), y el estudiante, valorando sus habilidades lingüísticas en ese idioma, manifiesta temores sobre su capacidad de abordar esta experiencia académica internacional con éxito.

Adicionalmente, el $2^{\circ}$ obstáculo apoyaría la tesis de que son necesarios cambios en el currículum de estos grados que incardinen, de forma más efectiva, la utilización del inglés como competencia transversal en un buen número de sus asignaturas. Dada la variabilidad de competencia en este idioma que muestran los estudiantes en primer año, no sería descartable el ofrecer un curso "0" previo a su llegada a la Universidad, o bien, una asignatura optativa en primer cuatrimestre del primer año, que contribuya a la nivelación de esos conocimientos, además de, alternativamente, ofrecer la posibilidad de certificarlos.

Tampoco debemos olvidar en este punto la importancia que tiene para la consecución de ese fin, por un lado, la mejora de la competencia lingüística de los profesores, y por otro, el reconocimiento en su carrera profesional de las actividades que realizan y que contribuyen a la movilidad internacional de los estudiantes, una dimensión importante ésta, de la internacionalización de la institución. Estas tareas pueden ser, tanto la orientación académica de estudiantes en los convenios Erasmus que tengan asignados, como la impartición de asignaturas en inglés. Ambas cuestiones se han puesto de manifiesto en el documento Estrategia para la Internacionalización de las Universidades Españolas 2015-20 (MECD, 2014).

Merece la pena señalar en este punto, y en relación al posible efecto positivo que una reorganización académica de los grados podría tener en la movilidad internacional, que un $51 \%$ de los estudiantes del grado S (38\% en el grado CCSS) señalaron como un obstáculo de relevancia "la existencia de asignaturas anuales cuando la estancia era semestral”. Esta dificultad también es percibida por los coordinadores Erasmus cuando tutelan alumnos Erasmus entrantes que, mayoritariamente en ambos grados, pasan en nuestro centro un único cuatrimestre (normalmente el $1^{\circ}$ ). Esa diferencia de relevancia entre los grados puede deberse a que, como se ha indicado anteriormente, los estudiantes $\mathrm{S}$ realizan más frecuentemente estancias semestrales que los de CCSS, porque la mayor parte de los acuerdos sólo permite estancias de esta duración.

Adicionalmente, se señalan en $3^{\circ}$ a $5^{\circ}$ lugar de importancia, obstáculos relacionados con el apoyo económico a la movilidad, que los estudiantes perciben como insuficiente, además de las dudas sobre el verdadero coste económico asociado con ella. La encuesta MOV_ES realizada en 2012 por Campus Vivendi (Observatorio de la Participación y Condiciones de Vida de los Estudiantes Universitarios Españoles, MECD), entre otros colectivos a estudiantes Erasmus salientes españoles, ponía de manifiesto que la beca recibida solo había cubierto el $40 \%$ de los gastos incurridos en la estancia de movilidad. Una vez más, mejorar la información que se proporciona sobre el coste de las estancias por país, contribuiría a reducir esa incertidumbre.

Es interesante constatar, que más allá de la importancia que le conceden al importe de la ayuda, consideren también relevante el conocer con antelación el momento en que se va a percibir. En los últimos años, los pagos de las becas Erasmus se han retrasado de forma importante, lo que sin duda habrá supuesto tensiones económicas adicionales en familias con bajos recursos económicos. De mantenerse esa situación, sólo aquellos estudiantes cuyo entorno familiar pueda sostenerlos durante toda la estancia podrían disfrutarla, lo que entraría en contradicción con la dimensión social de Erasmus, esto es, de la equidad e inclusividad que ha perseguido desde sus inicios en 1987 (Ariño, Soler \& Llopis, 2014).

Estos hallazgos subrayan la importancia de diseñar políticas de comunicación más activas y eficaces para el apoyo a la movilidad internacional, así como una voluntad política de los organismos financiadores de que las becas lleguen a los estudiantes antes de desplazarse a la universidad de acogida, de modo que cubran los elevados gastos a los que se enfrentan al inicio de la movilidad.

\section{AgRADECIMIENTOS}

A todos los estudiantes que respondieron a la encuesta de movilidad Erasmus y a sus coordinadores/responsable administrativo de movilidad en la Facultad de Ciencias de la Salud y del Deporte, de la Universidad de Zaragoza que la hicieron posible.

\section{REFERENCIAS}

Ariño, A., Soler I. \& Llopis R. (2014). La movilidad estudiantil universitaria en España. Revista de la Asociación de la Sociología de la Educación, 7 (1), 143167.

Briz-Ponce, L., Juanes-Méndez, J. A., \& García-Peñalvo, F. J. (2016). The Role of Gender in Technology Acceptance for Medical Education. En M. Cruz-Cunha, Miranda, I. Maria, R. Martinho \& R. Rijo (Coords), Encyclopedia of E-Health and Telemedicine (pp. 1013-1027). IGI Global. doi:10.4018/978-1-4666-9978-610.4018/978-1-46669978-6.ch079

European Higher Education Area (EHEA) Mobility Strategy (2012). Mobility for Better Learning. Mobility Strategy 2020 for the European Higher Education Area. Recuperado de: http://www.ehea.info/Uploads/(1)/2012 EHEA Mobility Strategy.pdf

Ministerio de Educación, Cultura y Deporte (MECD) (2014). Estrategia para la internacionalización de las universidades españolas 2015 - 2020. Recuperado de http://www.mecd.gob.es/educacion$\mathrm{mecd} / \mathrm{dms} / \mathrm{mecd} /$ educacion-mecd/areaseducacion/universidades/politicainternacional/estrategiainternacionalizacion/EstrategiaInternacionalizaci-nFinal.pdf

Ministerio de Educación, Cultura y Deporte (MECD) (2016). Datos y Cifras del Sistema Universitario Español. Curso 2015-2016. Recuperado de https://sede.educacion.gob.es/publiventa/datos-y-cifras- 
del-sistema-universitario-espanol-curso-

20152016/estadisticas-universidad-espana/21461

Souto-Otero, M., Huisman, J., Beerkens, M., de Win, H. \& Vujic, S. (2013). Barriers to international student mobility: evidence from the Erasmus program. Educational Researcher, 42(2), 70-77.
Teichler, U. (2004). Temporary study abroad: The life of ERASMUS students. European Journal of Education, 39(4), 395-408.

Vossensteyn, H., Beerkens, M., Cremonini, L., Huisman, J.,Souto-Otero, M., Bresancon, B., Focken, N., Leurs, B. McCoshan, A., Mozuraityte, N., Pimentel Bótas, P. C. \& de Wit, H. (2010). Improving participation in the Erasmus programme. Final report to the European Parliament. Recuperado de http://opus.bath.ac.uk/21174/ 